\title{
Colossal Magnetoresistance and Phase Separation in Manganite Thin Films
}

\author{
M. K. Srivastava ${ }^{1,3}$, V. Agarwal ${ }^{2}$, A. Kaur ${ }^{3}$, and H. K. Singh ${ }^{4}$ \\ ${ }^{I}$ Deshbandhu College, University of Delhi, Kalkaji, New Delhi-110019, India \\ ${ }^{2}$ Maitreyi College, University of Delhi, Chankyapuri, New Delhi-110021, India \\ ${ }^{3}$ Department of Physics and Astrophysics, University of Delhi, Delhi-110007, India \\ ${ }^{4}$ CSIR-National Physical Laboratory, Dr. K. S. Krishnan Marg, New Delhi-110012, India
}

\begin{abstract}
In the present work, polycrystalline $\mathrm{Sm}_{0.55} \mathrm{Sr}_{0.45} \mathrm{MnO}_{3}$ thin films were prepared on LSAT (001) single crystal substrates by ultrasonic nebulized spray pyrolysis technique. The X-ray diffraction $\theta-2 \theta$ scan reveals that these films (i) have very good crystallinity, (ii) are oriented along out-of-plane c-direction, and (iii) are under small tensile strain. The impact of oxygen vacancy results into (i) higher value of paramagnetic insulator (PMI) to ferromagnetic metal (FMM) transition temperature, i.e., $\mathrm{T}_{\mathrm{C}} / \mathrm{T}_{\mathrm{IM}}$, (ii) sharper PMI-FMM transition, (iii) higher value of magnetization and magnetic saturation moment, and (iv) higher value of magnetoresistance $(\sim 99 \%)$. We suggest here that oxygen vacancy favors FMM phase while oxygen vacancy annihilation leads to antiferromagnetic-charge ordered insulator (AFM-COI) phase. The observed results have been explained in context of phase separation (PS) caused by different fractions of the competing FMM and AFM-COI phases.
\end{abstract}

Keywords: Manganites, thin film, colossal magnetoresistance, phase separation.

PACS: 75.47.Lx, 73.50.-h, 75.47.Gk, 64.75.St

\section{INTRODUCTION}

The manganese oxides, widely known as doped rare earth manganites $\mathrm{RE}_{1-\mathrm{x}} \mathrm{AE}_{\mathrm{x}} \mathrm{MnO}_{3}$ (RE: rare earth cations, AE: alkaline earth cations), near half doping range, have been extensively investigated due to the huge occurrence of interesting phenomena, viz., colossal magnetoresistance (CMR), many magnetoelectronic phases, phase separation (PS)/phase coexistence, first order nature of phase transition, etc [1]. In this context, $\mathrm{Sm}_{1-\mathrm{x}} \mathrm{Sr}_{\mathrm{x}} \mathrm{MnO}_{3}$ has been recognized as a prototype system because of its proximity to the charge order/orbital order $(\mathrm{CO} / \mathrm{OO})$ instability, most abrupt insulator-metal transition (IMT) and exhibits huge magnetoresistance. CMR is observed at all the compositions that correspond to the FMM ground state in the range $0.3<x \leq 0.52$. In the vicinity of $x \approx 0.45$, a very sharp (first order) transition from PMI to the FMM state is observed in $\mathrm{Sm}_{0.55} \mathrm{Sr}_{0.45} \mathrm{MnO}_{3}$. Such abrupt (first-order) PMI-FMM transitions are one of the prerequisites of a material having large magnetocaloric effect (MCE) and hence have technological potential. Like other low bandwidth manganites, $\mathrm{Sm}_{1-\mathrm{x}} \mathrm{Sr}_{\mathrm{x}} \mathrm{MnO}_{3}$ has a natural tendency towards phase separation/phase coexistence that causes evolution of a strong metamagnetic component in the vicinity of half doping $[2,3]$. The strong PS result in metamagnetism that render the composition- temperature $(x-\mathrm{T})$ phase diagram extremely fragile visa-vis external perturbations. Consequently, even mild external stimuli such as electromagnetic field, pressure, lattice strain provided by the substrate, annealing, grain boundaries, lattice imperfections, cationic vacancies, oxygen vacancies, etc., could dramatically modify their physical properties $[2,3]$. Here, we report the impact of oxygen vacancy on high field magnetotransport in polycrystalline $\mathrm{Sm}_{0.55} \mathrm{Sr}_{0.45} \mathrm{MnO}_{3}$ thin films deposited on single-crystalline LSAT (001) substrates and annealed in air and oxygen. We have attributed it to the fact that oxygen vacancy leads to FMM while oxygen vacancy annihilation favors AFM-COI phase.

\section{Experimental Details}

Polycrystalline $\quad \mathrm{Sm}_{0.55} \mathrm{Sr}_{0.45} \mathrm{MnO}_{3}$ thin films (thickness $\sim 100 \mathrm{~nm}$ ) were deposited on LSAT (001) single crystal substrates by ultrasonic nebulized spray pyrolysis technique at substrate temperature $\mathrm{T}_{\mathrm{S}} \approx 200^{\circ} \mathrm{C}$. These as grown films were divided into two sets wherein one set was annealed in air (named as LSATA) and other in flowing oxygen (named as LSAT-O), both at same temperature $\mathrm{T}_{\mathrm{A}} \approx 1000^{\circ} \mathrm{C}$ for $12 \mathrm{hrs}$ followed by slow cooling $\left(4^{\circ} \mathrm{C} / \mathrm{min}\right)$ to room temperature. The structural analysis was done by X-ray diffraction (XRD) in $\theta-2 \theta$ geometry. The magnetic and transport measurements were carried out by PPMS 
(Quantum Design). The temperature and magnetic field dependent electrical resistivity and magnetoresistance were measured in the temperature range $5-300 \mathrm{~K}$ and the magnetic field $(\mathrm{H}=50 \mathrm{kOe})$.

\section{Result and Discussion}

The occurrence of only ( $00 \ell)$ peaks in the XRD data confirms the highly oriented and polycrystalline nature of the films. The out-of-plane lattice parameter of the LSAT-A and LSAT-O films are $3.819 \AA$ and $3.823 \AA$, respectively which are slightly smaller than the reported value $\mathrm{c}=3.83 \AA$ confirms the tensile strain.

The temperature dependent resistivity $(\rho-T)$ of both the films were measured at $\mathrm{H}=0$ and $50 \mathrm{kOe}$. The zero field resistivity data of the LSAT-A shows IMT at $\mathrm{T}_{\mathrm{IM}} \approx 130 \mathrm{~K}$ accompanied by sharp drop in resistivity nearly three orders of magnitude, while broader and lower $\mathrm{T}_{\mathrm{IM}} \approx 125 \mathrm{~K}$ has been observed for LSAT-O film. The resistivity of both the films measured in zero field show well-defined hysteretic behavior with respect to the thermal cycling and in the cooling cycle IMT occurs at lower temperature. The large enhancement in $\mathrm{T}_{\mathrm{IM}} \approx 184 \mathrm{~K}\left(\Delta \mathrm{T}_{\mathrm{IM}}=54 \mathrm{~K}\right)$ and $172 \mathrm{~K}\left(\Delta \mathrm{T}_{\mathrm{IM}}=47 \mathrm{~K}\right)$ has been found for LSAT-A and LSAT-O films, respectively when measured in $\mathrm{H}=50 \mathrm{kOe}$ magnetic field. A huge difference in peak value of resistivity in two different fields has been observed where the zero field resistivity is considerably reduced with the application of high magnetic field confirms the strong nature of phase separation in these films.

The temperature dependent MR is calculated from resistivity data of both the films. A broad but huge $\mathrm{MR} \approx 99.81 \%$ and $\approx 98.75 \%$ in the vicinity of $\mathrm{T}_{\mathrm{C}} / \mathrm{T}_{\mathrm{IM}}$ has been observed for LSAT-A and LSAT-O films, respectively and then decreases slowly. The MR at lowest measured temperature $(5 \mathrm{~K})$ is also high, i.e., $\sim 40 \%$ and $\sim 50 \%$ for LSAT-A and LSAT-O films, respectively. The occurrence of CMR over such broad temperature range suggests towards appreciable presence of AFM-COI cluster in this temperature range which transformed into FMM ones by the applied magnetic field. The occurrence of huge MR in these films could also be due to strong suppression of spin fluctuations by the magnetic field.

The magnetic field dependent isothermal magnetoresistance of the LSAT-A and LSAT-O films measured at several temperatures is presented in Fig. 1 and Fig. 2, respectively. At $\mathrm{T}=5 \mathrm{~K}$, the resistivity showed a very sharp decrease in the first field increasing cycle and in subsequent magnetic field cycles this initial was not recovered. This results in larger $\mathrm{MR} \approx 44 \%$ in LSAT-O film than LSAT-A film $(\mathrm{MR} \approx 29 \%$ ) at $\mathrm{H}=50 \mathrm{kOe}$. At $\mathrm{T}=50 \mathrm{~K}$, both films show strong irreversibility and MR reaches to $\sim 70 \%$ and 87
$\%$ for LSAT-A and LSAT-O films, respectively at $\mathrm{H}=50 \mathrm{kOe}$. This could be due to presence of nonequilibrium metamagnetic clusters which is generally attributed to the collapse of the AFM-COI state, that is the magnetic field induced AFM-COI to FMM transformation. Such features have been observed in several low BW manganites having either AFM-COI ground state or a having a phase separated regime with

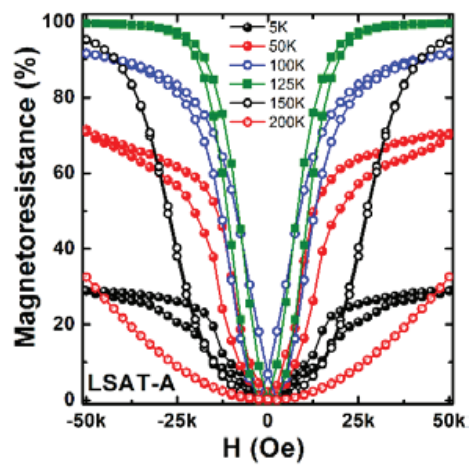

FIGURE 1: Variation of MR with magnetic field at different temperatures of LSAT-A film. competing FMM and AFM-COI components.

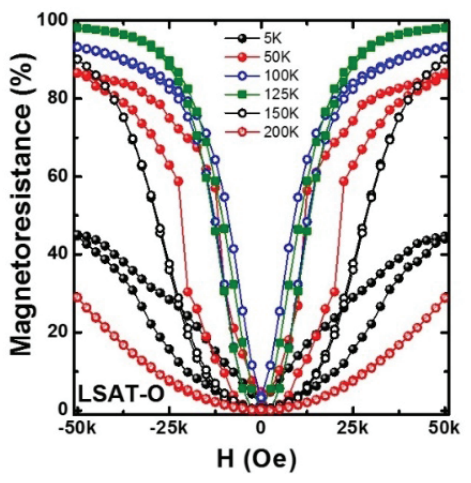

FIGURE 2: Variation of MR with magnetic field at different temperatures of LSAT-O film.

At $\mathrm{T}=100 \mathrm{~K}$ and $125 \mathrm{~K}$, hysteretic behavior remains same with narrower width, but at $125 \mathrm{~K}$, just below the $\mathrm{T}_{\mathrm{C}} / \mathrm{T}_{\mathrm{IM}}$ of LSAT-A film, a very sharp drop in resistivity followed by irreversibility in magnetic field cycling is observed. The MR calculated at $\mathrm{T}=125 \mathrm{~K}$ was found to be maximum $\approx 99.61 \%$ and $98.13 \%$ at $\mathrm{H}=50 \mathrm{kOe}$ with concomitant hysteresis for LSAT-A and LSAT-O films, respectively. At $\mathrm{T}=150 \mathrm{~K}$, the order of $\rho(\mathrm{H})$ drop is drastically reduced for both films. This behavior can be attributed to the sudden/quick transformation of COI cluster in the FMM phase. Large occurrence of MR 95\% (90\%) for LSAT-A (LSAT-O) well above $\mathrm{T}_{\mathrm{C}} / \mathrm{T}_{\mathrm{IM}}$ is very less studied in literature. At $\mathrm{T}=200 \mathrm{~K}$, i.e., in the PMI regime, the value of $\mathrm{MR}$ at $\mathrm{H}=50 \mathrm{kOe}$ for LSAT-A and LSAT-O films are $\approx 34 \%$ and $29 \%$, respectively. These observations show that the nature 
of the magnetic field induced transitions changes from the FOPT to second order phase transition (SOPT) around $\mathrm{T} \sim 150 \mathrm{~K}$. The occurrence of very large hysteretic MR even at moderate magnetic fields at $\mathrm{T}<\mathrm{T}_{\mathrm{C}}$ is clear signature of the presence of AFM-COI clusters in the FMM regime, while the presence of nonhysteretic but nonlinear $\mathrm{MR}$ at $\mathrm{T}>\mathrm{T}_{\mathrm{C}}$ shows the presence of AFM-COI clusters in the PMI regime.

In summary, PS and CMR ( 99\%) have been explained in terms of the variation of the relative fractions of the coexisting FM and AFM-CO phases as a function of the substrate induced strain and oxygen vacancy induced quenched disorder.

\section{ACKNOWLEDGMENTS}

MKS thanks CSIR for research fellowship.

\section{REFERENCES}

1. Y. Tokura, Rep. Prog. Phys. 69797 (2006).

2. M. K. Srivastava, P. K. Siwach, A. Kaur, and H. K. Singh, Appl. Phys. Lett. 97, 182503 (2010).

3. M. K. Srivastava, A. Kaur, K. K. Maurya, V. P. S. Awana, and H. K. Singh, Appl. Phys. Lett. 102, 032402 (2013). 